\title{
LOS HOSPEDEROS DE LAS PLANTAS HEMIPARÁSITAS DE LA FAMILIA LORANTHACEAE (s.l.) EN COSTA RICA
}

\author{
SILVIA LOBO C. \\ Herbario Nacional de Costa Rica (CR), Museo Nacional de Costa Rica \\ Apdo. postal 749-1000 San José, Costa Rica, A.C. museohn@ racsa.co.cr
}

\begin{abstract}
An actualized list of the hosts of the Costa Rican Loranthaceae (s.l.) species is presented. The information was obtained directly from the labels of the specimens deposited in the Herbario Nacional de Costa Rica (CR). The list includes 58 host families and 47 hemiparasite species.

RESUMEN. Se presenta una lista actualizada de los hospederos de las plantas hemiparásitas de la familia Loranthaceae (s.l.) en Costa Rica, con base en información obtenida directamente de los especímenes depositados en el Herbario Nacional de Costa Rica (CR). La lista incluye 58 familias de hospederos y 47 especies de hemiparásitas.
\end{abstract}

Key wORds / PALABRAS ClAve: Hemiparasite hosts, Loranthaceae (s.l.), Herbario Nacional de Costa Rica (CR), Costa Rica

A nivel mundial, aproximadamente 2500 especies de plantas floríferas, además de una gimnosperma, presentan algún grado de parasitismo (Gómez 1985). En Costa Rica, la mayoría de este tipo de plantas se encuentran representadas en la familia Loranthaceae (s.l.) por especies de arbustos o pequeños árboles hemiparásitos de ramas aéreas de árboles y arbustos (Burger \& Kuijt 1983). Una excepción a esta característica se presenta en el género Gaiadendron, que frecuentemente se encuentra como planta terrestre o parasitando las raíces del hospedero (Burger \& Kuijt 1983). Aunque todas estas especies están relacionadas entre sí, algunos autores las separan en las familias Loranthaceae (s.s.), Viscaceae y Eremolepidaceae (Burger \& Kuijt 1983). Además, existen en Costa Rica otras familias que presentan especies que parasitan las raíces (Scrophulariaceae, Hydnoraceae, Olacaceae, Orobanchaceae y Rafflesiaceae), los troncos (Rafflesiaceae), así como partes aéreas (Lauraceae) de árboles y arbustos.

Las especies costarricenses de la familia Loranthaceae (s.l.) parecen tener una amplia variedad de hospederos (Gómez 1985). A partir de la información de especímenes de herbario, Gómez (1985) anotó un total de 39 familias de hospederos de 38 especies de parásitas. También indicó que no se obtuvo el hospedero respectivo de 6 especies.

Aquí se presenta la lista de los hospederos de las plantas parásitas de la familia Loranthaceae (s.l.) en Costa Rica (Morales, inéd.), con base en la información de los especímenes depositados en el Herbario Nacional de Costa Rica (CR). Debido a que algunos especímenes presentan el nombre común del hospedero y no el nombre científico, éste se adaptó según León \& Poveda (1999).

De los 1273 ejemplares que se encontraban identificados hasta especie, un $32 \%$ cuenta con información sobre el hospedero; de este porcentaje sólo el $16 \%$ presenta información que puede confirmarse (por ej. una muestra del hospedero). La información sobre los hospederos se incluye mayormente a nivel de género (187 ejemplares), seguido por especie (110 ejemplares) y familia (72 ejemplares). En 40 ejemplares se indicaron los hospederos utilizando los nombres comunes. La lista abarca 47 especies de hemiparásitas, de las cuales 35 poseen información sobre el hospedero. Por otra parte, los hospederos pertenecen a 58 familias de dicotiledóneas, representadas por 123 géneros y 74 especies. De esta manera se agregan 24 familias más de hospederos a las 39 comunicadas por Gómez (1985), quien además menciona las familias Dilleniaceae, Pinaceae, Punicaceae y Saxifragaceae, mismas que no pudieron ser corroboradas. Según la revisión realizada, la especie de hospedero más representada es Guazuma ulmifolia (13 ejemplares), seguida por Theobroma cacao (11 ejemplares) y Matudaea trinervia (9 ejemplares); por otra parte, los géneros de hospederos más frecuentes son Quercus (33 ejemplares), Citrus (32 ejemplares) y Ficus (13 ejemplares). 
Los ejemplares de herbario son una fuente primaria de referencia, lo que hace que la información contenida en ellos sea de suma importancia. La poca referencia a los hospederos de las especies de la familia Loranthaceae (s.l.), observada en esta revisión, evidencia la necesidad de que los recolectores mejoren sus anotaciones sobre los especímenes y, en el caso particular de las especies hemiparásitas, sería recomendable recolectar también una muestra del hospedero. Esto ayudaría significativamente al estudio y entendimiento de la biología de las lorantáceas.

\section{LISTADELASESPECIES HEMIPARÁSITASDELAFAMILIA LORANTHACEAE (s.l.) YSUSRESPECTIVOSHOSPEDEROS, SEGÚNLAINFORMACIÓNDELOSEJEMPLARESDEPOSITADOS enel Herbario Nacionalde Costa Rica (CR)}

Especie hemiparásita

Antidaphne viscoidea Poepp. \& Endl.

\section{Hospedero}

Alnus acuminata, Ericaceae, Eugenia sp., Eugenia cf. guatemalensis, Ficus sp., Inga sp., Lauraceae, Matudaea trinervia, Moraceae, Myrtaceae, Psidium guajava, Quercus sp., Quercus copeyensis, Quercus seemannii

\section{Dendrophthora ambigua Kuijt}

Dendrophthora costaricensis Urb.
Brunellia sp., Quercus sp., Weinmannia pinnata

Cavendishia sp., Ericaceae, Escallonia myrtilloides, Macleania sp., Melastomataceae, Miconia sp., Miconia pittieri, Myrsine pit tieri, Myrtaceae, Pernettya sp., Quercus sp., Pentacalia firmipes, Solanaceae, Vaccinium sp.

Sin hospedero conocido

Calyptranthes sp., Ficus sp., Guarea sp., Melastomataceae, Symplococarpon sp.

Asteraceae, Cavendishia sp., Clethra sp., Ericaceae, Escallonia myrtilloides, Holodiscus argenteus, Myrsinaceae, Pernettya sp., Quercus sp., Ugni myricoides, Vaccinium sp., Vaccinium consan guineum

\section{Dendrophthora talamancana Kuijt}

Dendrophthora turrialbae Kuijt

Sin hospedero conocido

Gaiadendron punctatum (Ruiz \& Pav.) G. Don

Oryctanthus Eichler

Oryctanthus alveolatus (Kunth) Kuijt

Oryctanthus cordifolius (C. Presl) Urban

Oryctanthus occidentalis (L.) Eichler
Dendropanax querceti, Quercus sp.

Alchornea sp., Cornus disciflora, Escallonia sp., Myrcianthes rhopaloides, Myrsine pittieri, Quercus sp.

Chrysophyllum cainito

Abutilon sp., Bourreria oxyphylla, Calliandra sp., Theobroma cacao, Zanthoxylum sp.

Annonaceae, Boraginaceae, Burseraceae, Citrus sp., Cordia alliodora, Fabaceae, Guatteria sp., Hippocrateaceae, Hirtella americana, Paullinia sp.

Alchornea latifolia, Annonaceae, Citrus sp., Cnidoscolus aconiti folius, Coffea arabica, Ficus sp., Guatteria sp., Lauraceae, Myristica fragrans, Myrtaceae, Persea sp., Piper nigrum, Psidium guajava, Schlegelia sp., Theobroma cacao, Unonopsis sp., Viburnum sp., Virola sp., Vismia sp.

Alnus sp., Annona cherimola, Citrus sp., Cordia sp., Croton sp., Guatteria sp., Matudaea trinervia, Melastomataceae, Miconia sp., 


\begin{tabular}{|c|c|}
\hline Especie hemiparásita & Hospedero \\
\hline Oryctanthus spicatus (continúa) & $\begin{array}{l}\text { Pithecellobium sp., Quercus seemannii, Rutaceae, Syzygium } \\
\text { malaccense, Zanthoxylum sp. }\end{array}$ \\
\hline Panamanthus panamensis (Rizzini) Kuijt & Sin hospedero conocido \\
\hline Phoradendron Nutt. & Calliandra sp., Otoba sp., Solanum aphyodendron \\
\hline Phoradendron acinacifolium Eichler & Cordia alliodora \\
\hline Phoradendron annulatum Oliv. & Quercus sp. \\
\hline Phoradendron chrysocladon A. Gray & $\begin{array}{l}\text { Citrus sp., Conostegia sp., Guarea sp., Guettarda sp., Inga sp., } \\
\text { Hampea appendiculata, Matudaea trinervia, Melastomataceae, } \\
\text { Quercus sp., Tetrorchidium costaricense, Vismia sp. }\end{array}$ \\
\hline Phoradendron crassifolium (Pohl ex DC.) Eichler & Melastomataceae, Oryctanthus occidentalis \\
\hline Phoradendron dichotomum (Bert.) Krug \& Urb. & Sin hospedero conocido \\
\hline Phoradendron dipterum Eichler & Sin hospedero conocido \\
\hline Phoradendron nitens Kuijt & Sin hospedero conocido \\
\hline Phoradendron piperoides (Kunth) Trel. & $\begin{array}{l}\text { Artocarpus sp., Calophyllum sp., Castilla elastica, Cecropia sp., } \\
\text { Clusia sp., Croton niveus, Fabaceae, Ficus sp., Guarea sp., Inga } \\
\text { sp., Ocotea sp., Psidium guajava, Rubiaceae, Sloanea sp., } \\
\text { Theobroma cacao, Vochysia ferruginea }\end{array}$ \\
\hline $\begin{array}{l}\text { Phoradendron quadrangulare (Kunth) Krug \& } \\
\text { Urb. }\end{array}$ & $\begin{array}{l}\text { Amphitecna latifolia, Bougainvillea sp., Fabaceae, Guazuma } \\
\text { ulmifolia, Persea americana, Quercus sp., Swietenia } \\
\text { macrophylla, Tiliaceae }\end{array}$ \\
\hline Phoradendron robaloense Woodson ex Rizzini & Sapotaceae, Quercus corrugata \\
\hline Phoradendron robustissimum Eichler & $\begin{array}{l}\text { Cedrela sp., Cordia alliodora, Fabaceae, Inga sp., Lonchocarpus } \\
\text { cf. pentaphyllus, Muntingia calabura, Sapium sp., Sapium glan } \\
\text { dulosum, Symphonia globulifera, Tabebuia sp., Tiliaceae, Vitex sp. }\end{array}$ \\
\hline Phoradendron tonduzii Trel. & $\begin{array}{l}\text { Bunchosia veluticarpa, Cordia stellifera, Croton sp., Croton } \\
\text { draco, Erythrina berteroana, Malvaviscus sp., Melastomataceae, } \\
\text { Quercus sp., Sinclairia polyantha, Stevia sp., Styrax sp., } \\
\text { Trichilia sp. }\end{array}$ \\
\hline Phoradendron undulatum Eichler & $\begin{array}{l}\text { Conostegia xalapensis, Cordia alliodora, Croton sp., } \\
\text { Dendrophthora ambigua, Meliosma sp., Myrtaceae, Psidium sp., } \\
\text { Psidium guajava, Quercus corrugata }\end{array}$ \\
\hline Phoradendron woodsonii Trel. & Rubiaceae \\
\hline Phthirusa pyrifolia (Kunth) Eichler & $\begin{array}{l}\text { Calliandra sp., Cassia sp., Citrus sp., Citrus aurantium, Cordia } \\
\text { sp., Cordia alliodora, Coffea arabica, Fabaceae, Flacourtiaceae, } \\
\text { Guazuma ulmifolia, Inga sp., Myrtaceae, Oryctanthus occiden } \\
\text { talis, Psidium guajava, Senna reticulata, Spondias sp., } \\
\text { Theobroma sp., Vernonia patens, Zanthoxylum sp. }\end{array}$ \\
\hline Phthirusa stelis (L.) Kuijt & Trichilia havanensis \\
\hline Psittacanthus Mart. & Quercus sp. \\
\hline Psittacanthus corynocephalus Eichler & Sin hospedero conocido \\
\hline Psittacanthus costaricensis Kuijt & Sin hospedero conocido \\
\hline Psittacanthus cucullaris (Lam.) Blume & Sin hospedero conocido \\
\hline
\end{tabular}




\begin{tabular}{|c|c|}
\hline Especie hemiparásita & Hospedero \\
\hline Psittacanthus krameri Kuijt, ined. & $\begin{array}{l}\text { Erythroxylum sp., Nectandra membranacea, Pelliciera rhi } \\
\text { zophorae, Pimenta sp. }\end{array}$ \\
\hline Psittacanthus nodosus (Desr.) G. Don & Sin hospedero conocido \\
\hline Psittacanthus ramiflorus (DC.) G. Don & $\begin{array}{l}\text { Beilschmiedia sp., Inga sp., Lauraceae, Melastomataceae, } \\
\text { Psidium sp., Psidium guajava, Quercus sp., Quercus seemannii }\end{array}$ \\
\hline Psittacanthus rhynchanthus (Benth.) Kuijt & $\begin{array}{l}\text { Bursera simaruba, Burseraceae, Gliricidia sepium, Spondias } \\
\text { mombin, Spondias purpurea }\end{array}$ \\
\hline $\begin{array}{l}\text { Psittacanthus schiedeanus (Schltdl. \& Cham.) } \\
\text { Blume }\end{array}$ & $\begin{array}{l}\text { Alchornea sp., Alnus acuminata, Drimys granadensis, Myrtaceae, } \\
\text { Quercus sp., Quercus costaricensis }\end{array}$ \\
\hline Struthanthus Mart. & Symplocos sp. \\
\hline Struthanthus burgeri Kuijt & Sin hospedero conocido \\
\hline Struthanthus cansjeraefolius (Oliv.) Eichler & $\begin{array}{l}\text { Buddleja sp., Citrus sp., Cornuttia sp., Lippia torresii, } \\
\text { Monochaetum sp., Quercus sp., Quercus copeyensis, Solanaceae, } \\
\text { Theobroma sp. }\end{array}$ \\
\hline Struthanthus costaricensis Standl. & Citrus sp., Coffea arabica, Persea americana, Theobroma cacao \\
\hline Struthanthus hartwegii (Benth.) Standl. & Sin hospedero conocido \\
\hline Struthanthus leptostachyus (Kunth) G. Don & $\begin{array}{l}\text { Anaxagorea sp., Averrhoa carambola, Citrus sp., Cleyera } \\
\text { theioides, Eucalyptus sp., Ficus sp., Inga sp., Melastomataceae, } \\
\text { Pithecellobium sp., Rubiaceae, Syzygium sp., Theobroma cacao, } \\
\text { Trichospermum galeottii }\end{array}$ \\
\hline Struthanthus oerstedii (Oliv.) Standl. \& Calderón & $\begin{array}{l}\text { Avicennia sp., Citrus sp., Croton niveus, Gliricidia sepium, } \\
\text { Guarea sp., Laguncularia racemosa, Myrtus sp., Prunus persica, } \\
\text { Rutaceae, Salix babylonica, Zanthoxylum sp. }\end{array}$ \\
\hline Struthanthus orbicularis (Kunth) Blume & $\begin{array}{l}\text { Abutilon sp., Bunchosia sp., Citrus sp., Cnidoscolus aconitifolius, } \\
\text { Crescentia sp., Ericaceae, Erythrina sp., Ficus sp., Hibiscus sp., } \\
\text { Pithecellobium sp., Psidium sp., Robinsonella lindeniana, } \\
\text { Terminalia sp., Theobroma cacao }\end{array}$ \\
\hline $\begin{array}{l}\text { Struthanthus quercicola (Schltdl. \& Cham.) } \\
\text { Blume }\end{array}$ & $\begin{array}{l}\text { Meriania phlomoides, Coffea arabica, Coussarea sp., Ericaceae, } \\
\text { Fabaceae, Ficus sp., Guarea sp., Hedyosmum goudotianum, } \\
\text { Hedyosmum mexicanum, Macleania sp., Malus sylvestris } \\
\text { Melastomataceae, Miconia sp., Miconia tonduzii, Salix sp., } \\
\text { Solanaceae, Urera sp. }\end{array}$ \\
\hline Struthanthus woodsonii Cufod. & Rubiaceae \\
\hline
\end{tabular}

\section{AGRADECIMIENTOS}

Quisiera agradecer a Armando Ruiz B. (CR) por su colaboración en la revisión del presente manuscrito.

\section{Literatura Citada}

Burger, W. \& Kuijt, J. 1983. Loranthaceae (s.1.). p. 29-79. In: Burger, W. (ed.). Flora Costaricensis. Field Museum of Natural History. 255 p.

Gómez, L. D. 1985. Parasitic Plants. p. 292-298. In:
Janzen, D. (ed.). Costa Rican Natural History. The University of Chicago Press. 816 p.

León, J. \& Poveda, L. J. 1999. Nombres Comunes de las Plantas en Costa Rica. UNA, Heredia, Costa Rica. 870 p.

Morales, J. F. (inéd.). Loranthaceae. In: Grayum, M. \& Zamora, N. (eds.). 1998. Manual de Plantas de Costa Rica. Instituto Nacional de Biodiversidad, Heredia, Costa Rica. (manuscrito). 